\title{
ECG compression and labview implementation
}

\author{
Tatiparti Padma ${ }^{1}$, M. Madhavi Latha², Abrar Ahmed ${ }^{3}$ \\ ${ }^{1}$ GRIET, JNTU, Hyderabad, India, Member IETE; ${ }^{2}$ JNTU, Hyderabad, India, Member IEEE; ${ }^{3}$ GRIET, Hyderabad, India. \\ Email: tatipartipadma@gmail.com
}

Received 11 February 2009; revised 19 March 2009; accepted 25 March 2009.

\begin{abstract}
It is often very difficult for the patient to tell the difference between angina symptoms and heart attack symptoms, so it is very important to recognize the signs of heart attack and immediately seek medical attention. A practical case of this type of remote consultation is examined in this paper. To deal with the huge amount of electrocardiogram (ECG) data for analysis, storage and transmission; an efficient ECG compression technique is needed to reduce the amount of data as much as possible while preserving the clinical significant signal for cardiac diagnosis. Here the ECG signal is analyzed for various parameters such as heart rate, QRS-width, etc. Then the various parameters and the compressed signal can be transmitted with less channel capacity. Comparison of various ECG compression techniques like TURNING POINT, AZTEC, CORTES, FFT and DCT it was found that DCT is the best suitable compression technique with compression ratio of about 100:1. In addition, different techniques are available for implementation of hardware components for signal pickup the virtual implementation with labview is also used for analysis of various cardiac parameters and to identify the abnormalities like Tachycardia, Bradycardia, AV Block, etc. Both hardware and virtual implementation are also detailed in this context.
\end{abstract}

Keywords: ECG Compression; Labview; Implementation

\section{INTRODUCTION}

An electrocardiogram (ECG or EKG) is a recording of the electrical activity of the heart over time produced by an electrocardiograph. Electrical impulses in the heart originate in the sinoatrial node and travel through the heart muscle where they impart electrical initiation of systole or contraction of the heart. The electrical waves can be measured at selectively placed electrodes (electrical contacts) on the skin. Electrodes on different sides of the heart measure the activity of different parts of the heart muscle. An ECG displays the voltage between pairs of these electrodes, and the muscle activity that they measure, from different directions, also understood as vectors. After acquiring the signal, different signal analysis techniques using MATLAB software where various abnormalities can be traced out in ECG of a particular patient. The signal is then transmitted using wireless technology using Blue-Tooth as a transmitting technique. The device operates at a range of $100-150 \mathrm{~m}$, a distance that is ideal for use in a hospital.

Digital analysis of electrocardiogram (ECG) signal imposes a practical requirement that digitized data be selectively compressed to minimize analysis efforts and data storage space. Therefore, it is desirable to carry out data reduction or data compression. Data reduction is achieved by discarding digitized samples that are not important for subsequent pattern analysis and rhythm interpretation. Examples of such data reduction algorithms are: AZTEC, turning point (TP). AZTEC retains only the samples for which there is sufficient amplitude change. TP retains points where the signal curves (such as at the QRS peak) and discards every alternate sample. The data reduction algorithms are empirically designed to achieve good reduction without causing significant distortion error.

Another class of algorithms compresses the data under mathematically rigorous rules, so that digitized samples are compressed and recovered under some reversible mathematical criteria operating under predefined error limits. This approach has the benefit that the original signal can be recovered by with a minimum loss of information.

Einthoven named the waves he observed on the ECG using five capital letters from the alphabet: P, Q, R, S, and $T$. The width of a wave on the horizontal axis represents a measure of time. The height and depth of a wave represent a measure of voltage. An upward deflection of a wave is called positive deflection and a downward deflection is called negative deflection. A typical representation of the ECG waves is presented in the following Figure 1. 


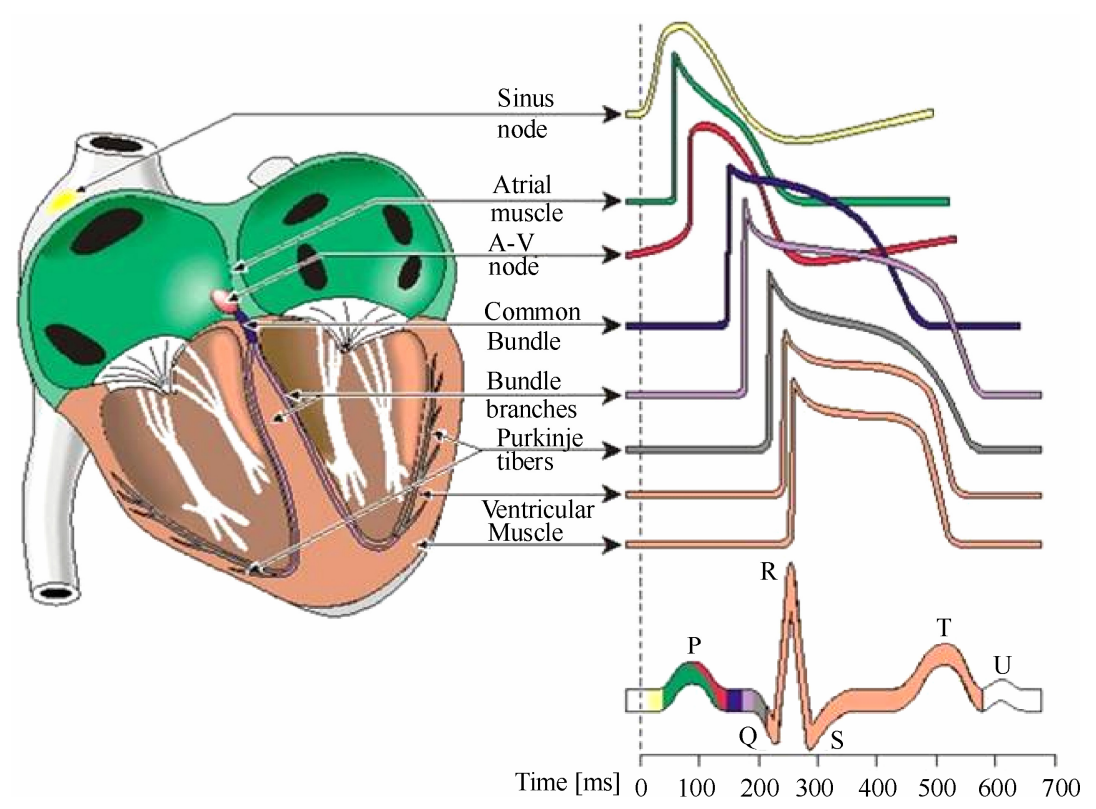

Figure 1. A typical representation of the ECG waves.

\section{SYSTEM DESCRIPTION}

Continuous monitoring of the electrocardiogram in both inpatients and ambulatory subjects has become a very common procedure during the past thirty years, with diverse applications ranging from screening for cardiac arrhythmias or transient ischemia, to evaluation of the efficacy of anti arrhythmic drug therapy, to surgical and critical care monitoring. The need for automated data reduction and analysis of the ECG has been apparent, motivated by the very large amount of data that must be analyzed (on the order of $10^{5}$ cardiac cycles per patient per day). As clinical experience has led to the identification of more and more prognostic indicators in the ECG, clinicians have demanded and received increasingly sophisticated automated ECG analyzers.

Visual analysis of the ECG is far from simple. Accurate diagnosis of ECG abnormalities requires attention to subtle features of the signals, features that may appear only rarely, and which are often obscured or mimicked by noise. Diagnostic criteria are complicated by inter- and intra-patient variability of both normal and abnormal ECG features. In this paper, the attempt is made to replace the bedside monitors in the intensive care units so as to reduce the workload of the staff and increase the efficiency in interpreting the abnormalities. The basic block diagram of the module is as shown in the Figure 2.

The standard lead system used in intensive care units is lead II system; the acquired signal is taken and is fed to an instrumentation amplifier that amplifies the signal. The amplifier is used to set the gain and it also amplifies very low amplitude ECG signal into perceptible view.

The acquisition of pure ECG signal is of higher importance. As we know that the ECG signal will be in the range of milli-volts range, which is difficult to analyze. So the prior requirement is to amplify the acquired signal. The acquisition and amplification of ECG signal is showed in Figure 3 using an instrumentation amplifier AD620.

The output gain can be programmed by varying the value of $\mathrm{R}_{\mathrm{G}}$. The amplified output is shown in Figure 4 .

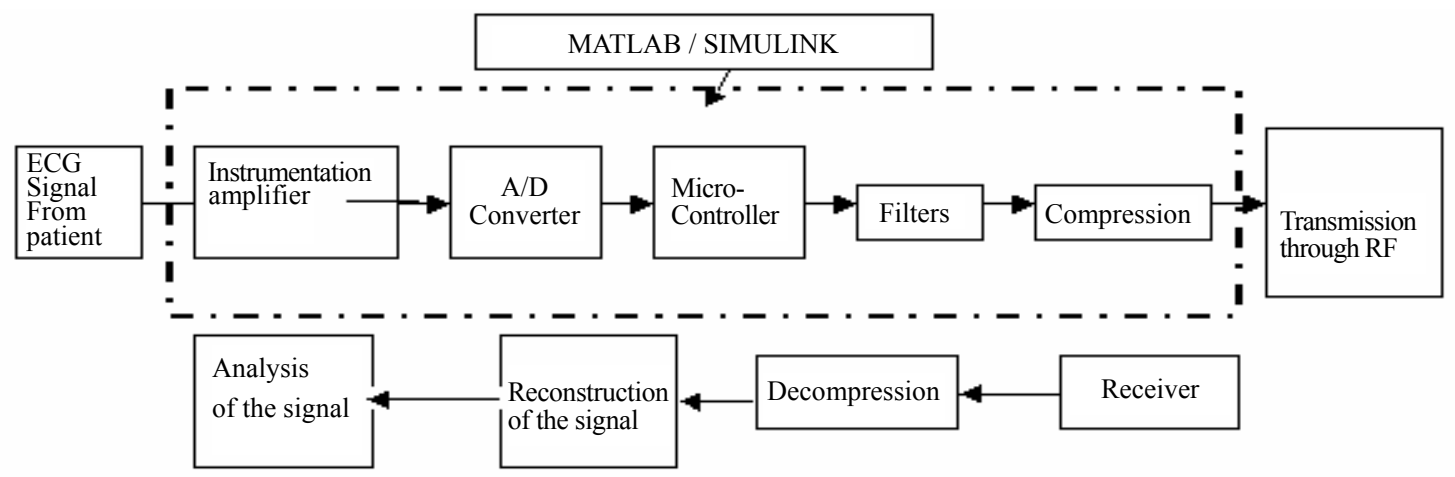

Figure 2. Basic block diagram of the ECG module. 


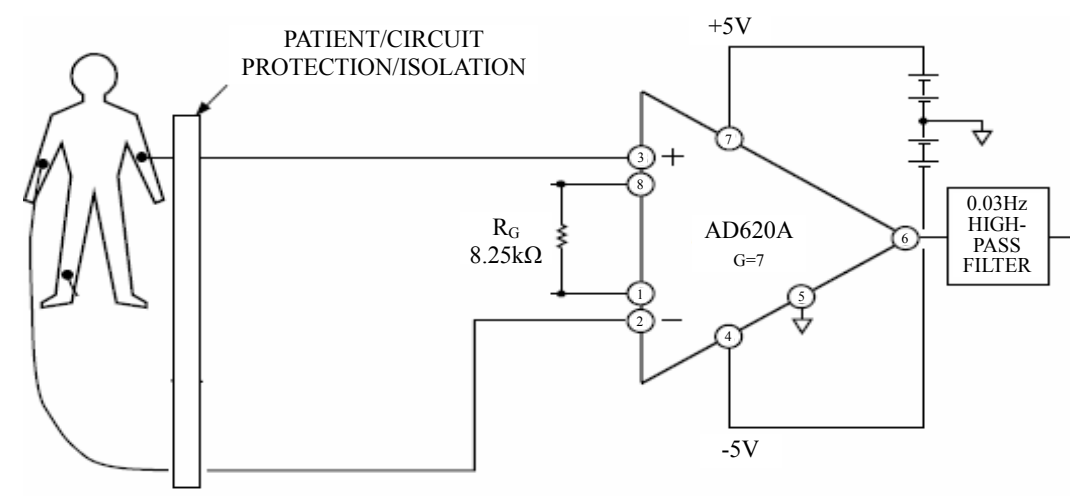

Figure 3. ECG acquisition with instrumentation amplifier AD620.

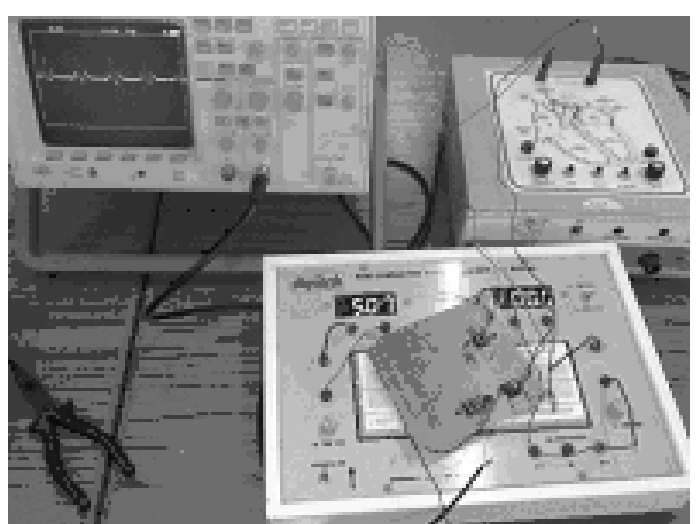

Figure 4. ECG signal output before ADC.

The amplified output is then fed to the analog to digital converter for digitalizing the ECG data using ADC and microcontroller. In this process, micro-controller is used so as to set the clocks for picking up the summation of the signals that are generated form the heart. The heart generates different signals at various nodes that is shown in Figure 1. The summation of the signals that are generated by the heart is taken and then it is sent for filtering processes.

The digital output of the ECG is displayed in LCD as shown in Figure 5.

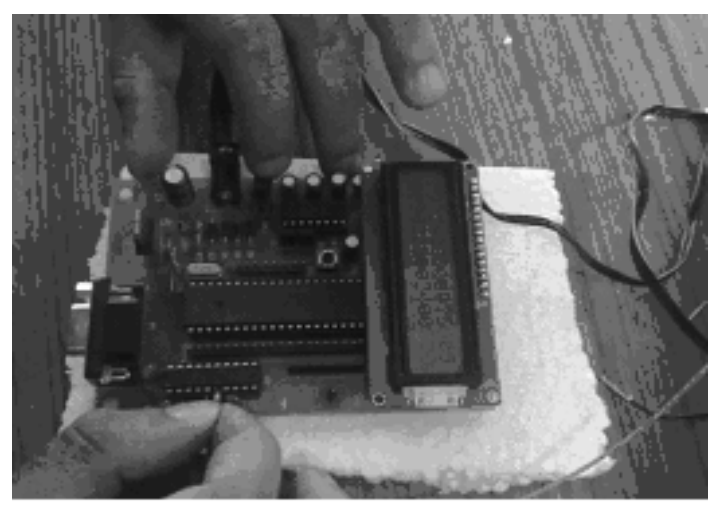

Figure 5. Digital output of the ECG is displayed in LCD.
As the ECG signal is going to be transmitted through wires to the module, it is obviously corrupted by various noises such as power line interference, muscle tremors etc. Hence various filtering techniques are applied to remove the noise and to send the error/noise free signal for further processing. Here adaptive noise filtering is used for removal of $50 \mathrm{~Hz}$ that is the power line interference because, the ECG signal also contains $50 \mathrm{~Hz}$ signal and if normal band reject filter is used, then the 50 $\mathrm{Hz}$ signal which is very important in the ECG signal will be lost. Therefore by opting adaptive noise filtering, the power line frequency can be eliminated at the same time retaining the $50 \mathrm{~Hz}$ signal in the original waveform.

After the filtering process, the signal is set for the transmission, but it is important to compress it so as to transmit at a faster rate. In this paper compared various basic compression techniques like Turning point, AZTEC, CORTES and found that CORTES is the better option for the compression of ECG signal as it compresses the signal at a rate of around 100:1. Before transmitting the compressed data, the ECG signal is analyzed.

\section{ECG ANALYSIS}

The processing and the analysis of the ECG has gained clinical significance. The various cardiac parameters are heart rate, R-R interval, QRS duration; etc can be obtained at any instance of time or continuously depending upon the requirement. The better analysis of the ECG can help doctors to give the appropriate care to the patients and also helps to avoid various severe situations that may arise. Here the ECG signal is analyzed and the result has been displayed as shown in Figure 6.

After analysis of ECG signal, it can be compressed using various techniques and hence transmit the compressed data to the main system. The various compression techniques have been explained below.

\section{COMPRESSION TECHNIQUES}

The various compression techniques like AZTEC, TP, CORTES, DFT, FFT algorithms are compared with PRD and Compression ratio and best suitable was considered. 


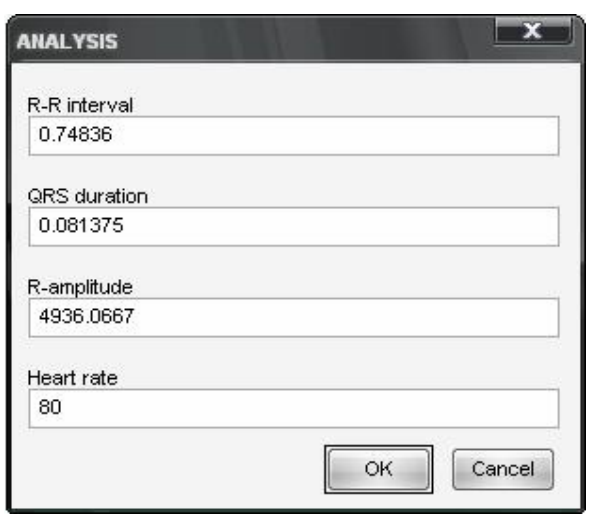

Figure 6. ECG analysis output.

\subsection{Turning Point Algorithm}

1) Acquire the ECG signal

2) Take the first three samples and check for the condition as mentioned below:

$$
\begin{gathered}
\left(\mathrm{x}_{1}-\mathrm{x}_{0}\right) *\left(\mathrm{x}_{2}-\mathrm{x}_{1}\right)<0 \\
(\text { or) } \\
\left(\mathrm{x}_{1}-\mathrm{x}_{0}\right) *\left(\mathrm{x}_{2}-\mathrm{x}_{1}\right)>0
\end{gathered}
$$

3) If the above condition- 1 is correct then $x_{1}$ is stored else $\mathrm{x}_{2}$ is stored.

4) Reconstructing the compressed signal.

The compression ratio of Turning point algorithm is $2: 1$, if higher compression is required then the same algorithm can be implemented on the already compressed signal so that it is further compressed to a ratio of $4: 1$. But after the $2^{\text {nd }}$ compression, the required data in the signal may be lost since the signal is overlapped on one another. Therefore, TP algorithm is limited to compression ratio of 2:1. TP algorithm can be applied on the

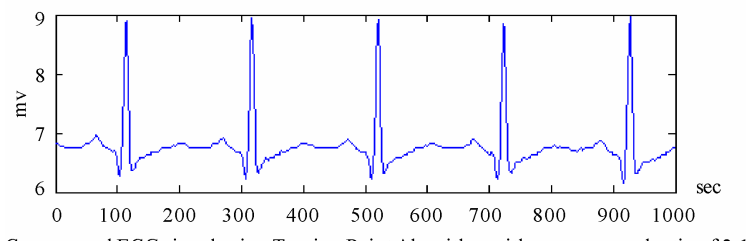

Compressed ECG signal using Turning Point Algorithm with a compressed ratio of 2:1

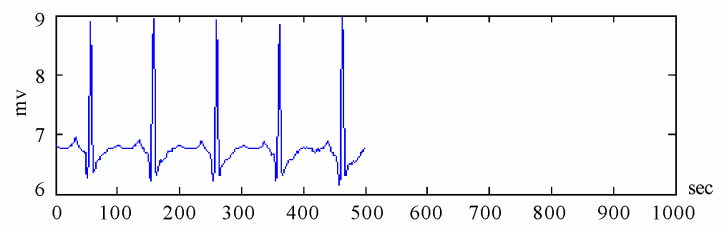

Compressed ECG signal using Turning Point Algorithm with a compressed ratio of 4:1

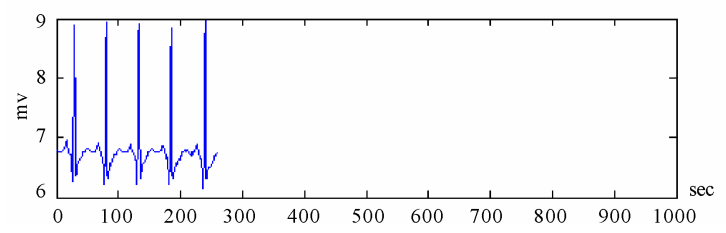

Figure 7. Turning point compression analysis. already compressed data to increase the compression ratio to 4:1. As shown in Figure 7.

\subsection{AZTEC ALGORITHM}

Another commonly used technique is known as AZTEC (Amplitude Zone Time Epoch Coding). This converts the ECG waveform into plateaus (flat line segments) and sloping lines. As there may be two consecutive plateaus at different heights, the reconstructed waveform shows discontinuities. Even though the AZTEC provides a high data reduction ratio, the fidelity of the reconstructed signal is not acceptable to the cardiologist because of the discontinuity (step-like quantization) that occurs in the reconstructed ECG waveform. As shown in Figure 8.

AZTEC Algorithm is implemented in 2 phases:

\subsubsection{Horizontal Mode}

1) Acquire the ECG signal

2) Assign the first sample to $X_{\max }$ and $X_{\min }$ which represents highest and lowest elevations of the current line.

3) Check for the following condition and store the plateau if

a) If $X_{1}>X_{\max }$ then $X_{\max }=X_{1}$ and

b) If $X_{1}<X_{\min }$ then $X_{\min }=X_{1}$ and so on till $X_{n}$ samples, repeat this until the following 2 conditions are satisfied

(1) the difference between VMAX and VMIN is greater than a predetermined threshold or

(2) if line length is $>50$ are satisfied

4) The stored values are the length $L=S-1$, where $S$ is no. of samples and $\mathrm{L}$ is length and the average amplitude of the plateau (VMAX+VMIN)/2.

5) Algorithm starts assigning the next samples to $X_{\max }$ and $\mathrm{X}_{\min }$.

\subsubsection{Slope Mode}

1) If no. of samples $<=3$, then the line parameters are not saved. Instead the algorithm begins to produce slopes.

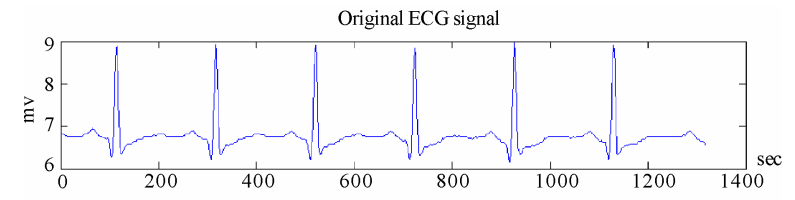

Compressed ECG signal using Turning Point Algorithm with a compressed ratio of 10:1
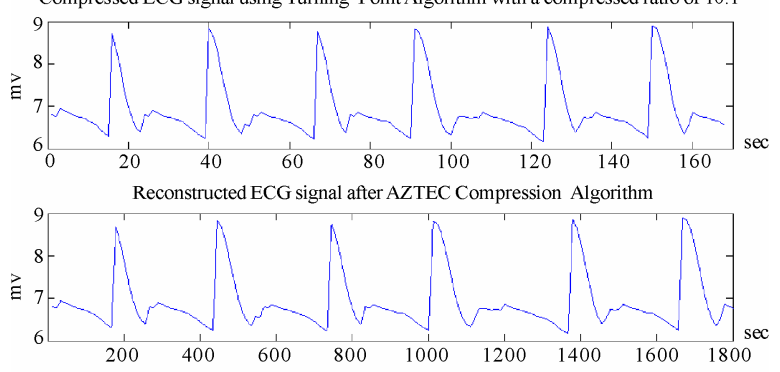

Figure 8. AZTEC compression analysis. 
2) The direction of the slope is determined by checking the following conditions.

a) If $\left(X_{2}-X_{1}\right) *\left(X_{1}-X_{0}\right)$ is +ve then the slope is +ve.

b) If $\left(\mathrm{X}_{2}-\mathrm{X}_{1}\right) *\left(\mathrm{X}_{1}-\mathrm{X}_{0}\right)$ is -ve then the slope is -ve.

3) The slope is terminated if the no. of samples is $>=3$ and if direction of slope is changed.

\subsection{CORTES Algorithm}

An enhanced method known as CORTES (Coordinate Reduction Time Encoding System) applies TP to some portions of the waveform and AZTEC to other portions and does not suffer from discontinuities. AZTEC line length threshold Lth, CORTES saves the AZTEC line otherwise it saves the TP data. As shown in Figure 9.

1) Acquire the ECG signal

2) Define the Vth and Lth.

3) Find the current Maximum and minimum.

4) If the Sample greater than threshold than compare the length with Lth

5) If (len>lth)

AZTEC Else

TP

6) Plot the compressed signal

\subsection{DCT Compression}

1) Separate the ECG components into three components $\mathrm{x}, \mathrm{y}, \mathrm{z}$.

2) Find the frequency and time between two samples.

3) Find the dct of ecg signal check for dct coefficients (before compression) $=0$, increment the counter $\mathrm{A}$ if it is between +0.22 to -0.22 and assign to Index $=0$.

4) Check for DCT coefficients(after compression) $=0$, increment the Counter B.

5) Calculate inverse det and plot decompression, error.

6) Calculate the compression ratio, PRD.

As shown in Figure 10.

\subsection{FFT Compression}

1) Separate the ECG components into three components $\mathrm{x}, \mathrm{y}, \mathrm{z}$.
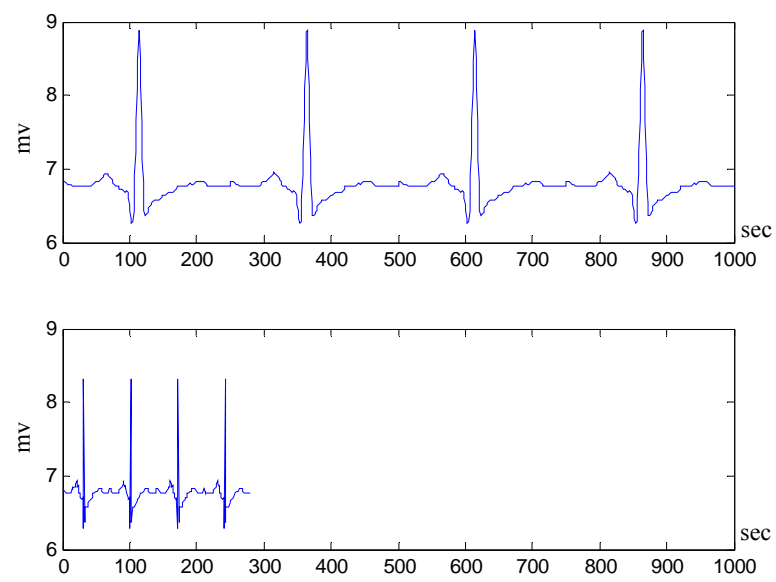

Figure 9. CORTES compression analysis.

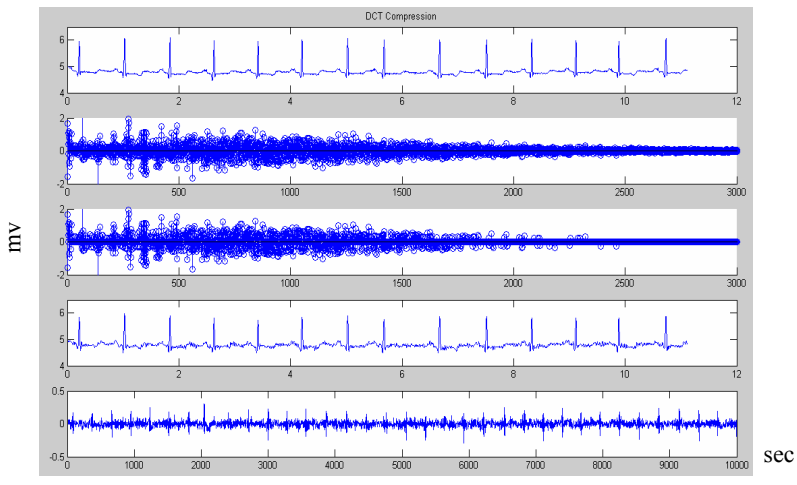

Figure 10. DCT compression analysis.

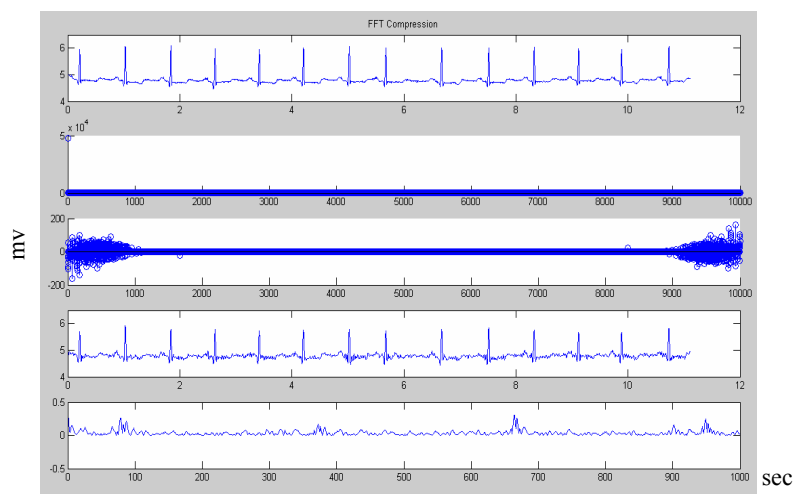

Figure 11. FFT compression analysis.

2) Find the frequency and time between two samples.

3) Find the FFT of ECG signal check for fft coefficients (before compression) $=0$, increment the counter $\mathrm{A}$ if it is between +25 to- 25 and assign to Index $=0$.

4) Check for FFT coefficients (after compression) $=0$, increment the Counter B.

5) Calculate inverse FFT and plot decompression, error.

6) Calculate the compression ratio, PRD.

As shown in Figure 11.

\subsection{Summary}

Summary of ECG data compression schemes.

The comparison table shown in Table 1 above, details the resultant compression techniques. This gives the choice to select the best suitable compression method. Hence in this project the DCT found to be compressed 90.43 with PRD as 0.93 .

Table 1. Comparison of compression techniques.

\begin{tabular}{lcc}
\hline METHOD & COMPRESSION RATIO & PRD \\
\hline CORTES & 4.8 & 3.75 \\
TURNING POINT & 5 & 3.20 \\
AZTEC & 10.37 & 2.42 \\
FFT & 89.57 & 1.16 \\
DCT & 90.43 & 0.93 \\
\hline
\end{tabular}




\section{HARDWARE IMPLEMENTATION}

The hardware implementation part is rather large and complex and the present trend in the BIOMEDICAL field is moving towards the miniaturization, thereby an efficient design flow is necessary, which was implemented using LABVIEW as shown in Figure 12.

The ECG signal is acquired with the help of electrodes that are connected to the patient and the signal is fed for further processing like instrumentation amplifier, Analog to Digital converter, micro controller, and filters. After acquiring the signal, different signal analysis techniques using LABVIEW software where various abnormalities are to be checked for and finally display the problem in ECG of a particular patient.

The standard lead system used in intensive care units is lead II system. (ECG data was acquired from a data file MIT-BIH (.m file)).

The signal is taken and fed to an instrumentation amplifier that amplifies the signal. The amplifier is used to set the gain and it also amplifies very low amplitude ECG signal into perceptible view. Then the signal goes for analog to digital conversion for the sake of easier transmission. The amplified signals are then sent for filtering processes.

The adaptive noise filtering is used for removal of 50 $\mathrm{Hz}$ that is the power line interference because, the ECG signal also contains $50 \mathrm{~Hz}$ signal and if normal band reject filter is used, then the $50 \mathrm{~Hz}$ signal which is very important in the ECG signal will be lost. Therefore by opting adaptive noise filtering, the power line frequency can be eliminated at the same time retaining the $50 \mathrm{~Hz}$ signal in the original waveform.

\section{RESULTS}

Extracting the portion of the signal and finding the $\mathrm{R}$ peaks in the signal by a first difference method. Once the $\mathrm{R}$ peaks are identified the heart rate is calculated the by knowing the period between successive Rpeaks.

$$
\mathrm{Y}(\mathrm{n})=\frac{\mathrm{x}(\mathrm{n}+1)-\mathrm{x}(\mathrm{n})}{\mathrm{T}} \text {; where } \mathrm{T} \text { is sampling period. }
$$

\section{Heart rate Calculation:}

$$
\mathrm{HR}=\frac{60}{\mathrm{Y}(\mathrm{n})}==
$$

\section{CONCLUSIONS}

The feeling of being in virtual contact with the health care professionals provides a sense of safety to the subjects, without the hassles of permanent monitoring.

Offers a valuable tool for easy measurement of ECG.

Offers first hand help when ever patient requires immediate medical attention.

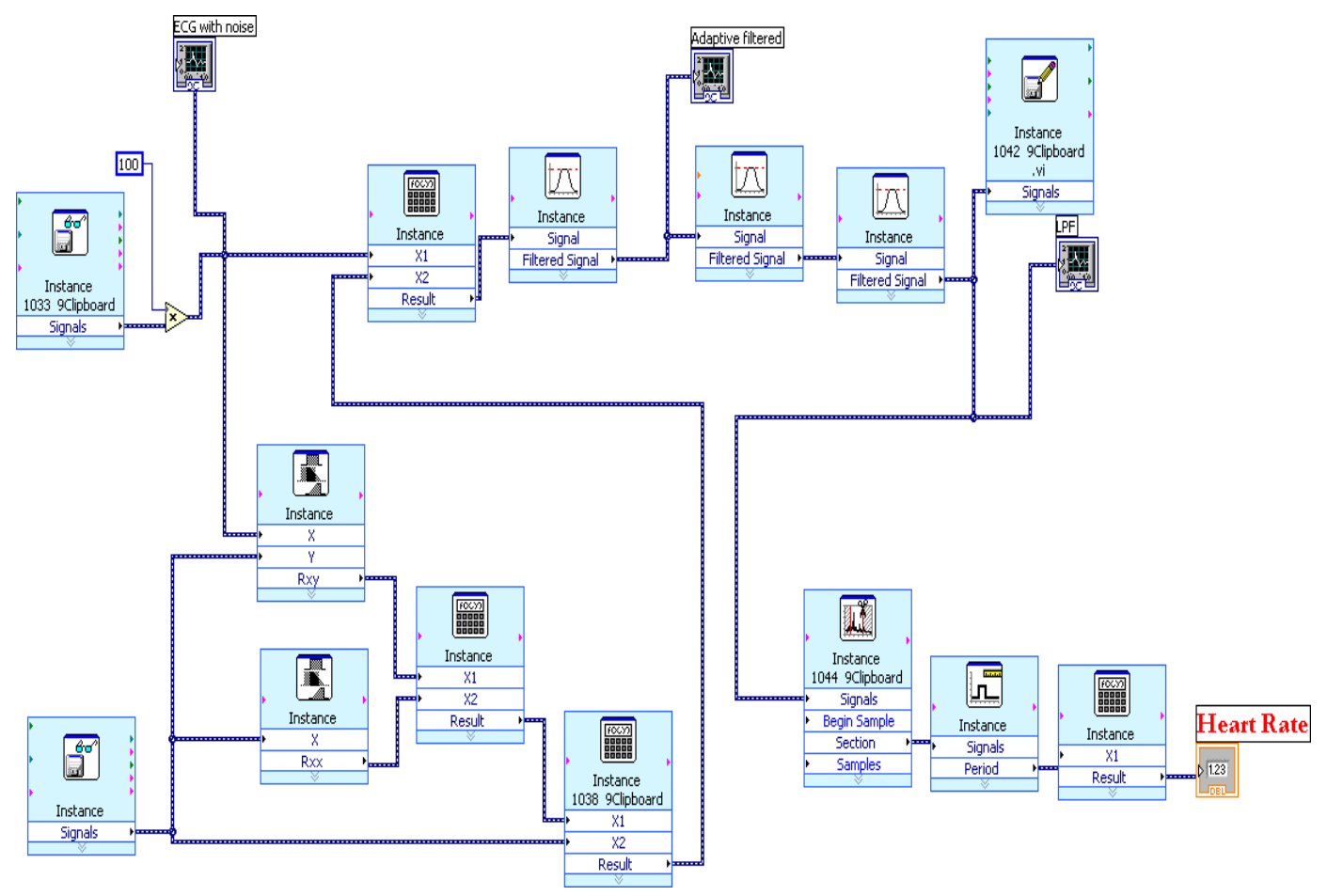

Figure 12. QRS detection \& heart rate calculation module. 


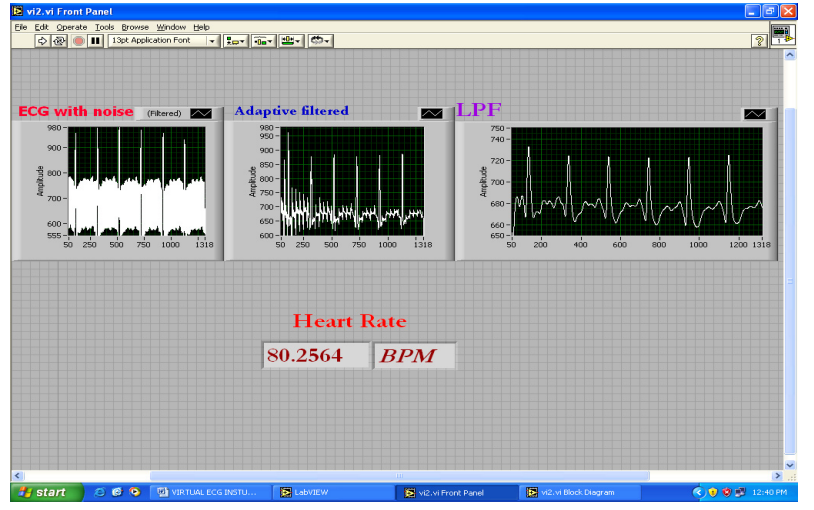

Figure 13. Heart rate display.

The results achieved were quite satisfactory the DCT found to be compressed 90.43 with PRD as 0.93. The signal analysis techniques using LABVIEW software where various abnormalities are to be checked for and finally display the problem in ECG of a particular patient was also given a positive indication, with this as the goal set the further implementation in matlab simulink work is also under the implementation stage.

\section{REFERENCES}

[1] S. Jalaleddine, C. Hutchens, R. Stratan, and W. A. Coberly, (1990) ECG data compression techniques-a unified approach, IEEE Trans. Biomed. Eng., 37, 329-343.

[2] J. R. Cox, F. M. Nolle, H. A. Fozzard, and G. C. Oliver, (1968) AZTEC, a preprocessing program for real time ECG rhythm analysis, IEEE Trans. Biomed. Eng., BME-15, 129-129.

[3] B. R. S. Reddy and I. S. N. Murthy, (1986) ECG data compression using fourier descriptors, IEEE Trans. Biomed. Eng., BME-33, 428-433.

[4] D. C. Reddy, (2007) Biomedical signal processing-principles and techniques, 254-300, Tata McGraw-Hill, Third reprint.

[5] J. L. Simmlow, Biosignal and biomedical image processing- MATLAB based applications, 4-29.

[6] A. S. Al-Fahoum, (2006) Quality assessment of ECG compression techniques using a wavelet-based diagnostic measure, IEEE Trans. in Biomedicine, 10, 182-191.

[7] V. Kumar, S. C. Saxena, and V. K. Giri, (2006) Direct data compression of ECG signal for telemedicine, ICSS , 10, $45-63$.

[8] A. Perkusich, G. S. Deep, M. L. B. Perkusich, and M. L. Varani, (1989) An expert ECG acquisition and analysis system, IMTC- 89, 184-189. 